\title{
The Effects of Episode Similarity on Children's Reports of a Repeated Event
}

\author{
Meaghan C. Danby ${ }^{\mathrm{a}}$, Stefanie J. Sharman ${ }^{\mathrm{a} *}$, Sonja P. Brubacher ${ }^{\mathrm{b}}$, Martine B. Powell ${ }^{\mathrm{b}}$ \\ ${ }^{\mathrm{a}}$ School of Psychology, Deakin University \\ ${ }^{\mathrm{b}}$ Centre for Investigative Interviewing, Griffith Criminology Institute, Griffith University
}

Preprint of: Danby, M. C., Sharman, S. J., Brubacher, S. P., \& Powell, M. B. (2019). The effects of episode similarity on children's reports of a repeated event. Memory, 27, 561 567. doi: 10.1080/09658211.2018.1529798

*Correspondence and reprint requests should be addressed to Stefanie Sharman, School of Psychology, Deakin University, 221 Burwood Highway, Burwood, VIC 3125, Australia (email: stefanie.sharman@ deakin.edu.au). 


\begin{abstract}
Much research has tested techniques to improve children's reporting of episodes from a repeated event by interviewing children after they have experienced multiple episodes of a scripted event. However, these studies have not considered any effects of the similarity shared between event episodes on children's reports. In the current study, 5- to 9-year-olds experienced four episodes of a scripted repeated event that shared a high $(n=76)$ or low $(n$ $=76$ ) degree of similarity, and were subsequently interviewed about individual episodes. The proportional amount and accuracy of children's reported details were tallied. Children reported proportionally more details and more script deviations after experiencing the high, compared to low, similarity event. Conversely, children were more accurate in their episodic reports when they experienced the low, compared to high, similarity event. The current findings have implications for the generalisability and comparability of past results across laboratory studies.
\end{abstract}

Keywords: children, repeated event, script theory, source monitoring, child interviewing 


\section{The Effects of Episode Similarity on Children's Reports of a Repeated Event}

Understanding children's memory for episodes from repeated events has interested researchers and interviewers for the past few decades (see Brubacher, Powell, \& Roberts, 2014, for a review). This work has been essential for assisting children to describe individual episodes of repeated offences, such as long-term abuse, in reasonable detail (that is, for children to particularise episodes; Powell, Roberts, \& Guadagno, 2007; $S$ v. $R, 1989$ ). With ongoing experience at an event, children become proficient at recognising the details that are typical across episodes, but they less accurately report the details that varied (Powell \& Thomson, 1996; Powell, Roberts, Ceci, \& Hembrooke, 1999; Connolly \& Lindsay, 2001). Extant experimental research has examined children's reports of repeated events comprising different ratios of typically-occurring and varying details. It is not yet known whether (and how) the composition of a repeated event impacts children's reporting of individual episodes.

Theoretically, children's reports of a repeated event would be expected to differ as a result of the details comprising the event. With repeated experience, children develop scripts that comprise slots for the typically-occurring objects and actions (Fivush, 1984; Schank \& Abelson, 1977). Scripts facilitate recall of what usually happens rather than what happened on an individual episode (Hudson \& Nelson, 1986; Schank \& Abelson, 1977). Scripts can be expected to develop at different rates depending on the degree of distinctiveness across episodes (see Hudson, Fivush, \& Kuebli, 1992, for a review). According to the schema-confirmation-deployment model, all details are prospective script components initially. With increasing experience at the event, the typically-occurring elements are "confirmed" as script components and the script is then "deployed" (Farrar \& 
Goodman, 1992). Scripts are confirmed and deployed more quickly when event episodes share a high degree of similarity to each other (Hudson et al., 1992). Once a script is deployed, children have more cognitive resources available to recognise details that deviate from the script (Farrar \& Goodman, 1992).

Typically, researchers examining children's memory for repeated events have engaged children in three to six event episodes that share a parallel structure (e.g., classroom activities or birthday parties conducted in the same temporal order each episode; Brubacher, Glisic, Roberts, \& Powell, 2011; Odegard, Cooper, Lampien, Reyna, \& Brainerd, 2009). The events contain critical to-be-remembered details about which children are questioned in post-event interviews. Sometimes details are presented identically in each episode (fixed; e.g., children always wear the same badge), sometimes details differ in each episode (variable; e.g., children always complete a different puzzle), and sometimes details are presented in only a single episode (new; e.g., activity leader wears a cape once only). Fixed details are expected to become incorporated in children's event script. Variable details are represented in scripts at two different levels: the broader items (e.g., completing a puzzle) occur as regularly as fixed details and are expected to become incorporated as slots, while the particular instantiations (e.g., the different puzzle designs) fill these slots (see Roberts, 2002). Children's scripts assist them to report the regularly-occurring details from an event (i.e., fixed details and variable items) proficiently. New details do not have a place in an event script as they do not typically occur.

The similarity that helps children to describe the typical experience of a repeated event makes the task of accurately discriminating among the episodes challenging (Lindsay, Johnson, \& Kwon, 1991; Powell et al., 1999; Powell \& Thomson, 1996; Powell, 
Thomson, \& Dietze, 1997). At retrieval, inferences are made about what happened during an episode by reconstructing the details from the script (Slackman \& Nelson, 1984), sometimes resulting in recall of details that are erroneous but script-consistent. For example, children commonly confuse varying instantiations across episodes (e.g., saying that the puzzle was a clown on a tightrope in the third episode when that puzzle was actually from the second episode) (Roberts \& Powell, 2001).

According to the source-monitoring framework, people engage in decision-making processes at retrieval about the origin (or "source") of recalled information (i.e., deciding which details were present in which episode; Johnson, Hashtroudi, \& Lindsay, 1993). This process is more challenging when sources are highly similar (Lindsay et al., 1991; Roberts, 2002; Thierry \& Pipe, 2009), and for children more so than adults (Roberts, 2002). In an examination of the impacts of similarity, Lindsay and colleagues (Experiment 2) had 4- and 6-year-olds and adults watch two videos depicting someone telling a story about a circus. The storytellers were perceptually very similar or dissimilar (in gender, age, and voice). Each storyteller described circus acts unique to their story, and acts that were common across stories but had different details. Young children were particularly likely to confuse who said what when the storytellers were similar, whereas older children and adults monitored the highly similar sources more effectively. When sources were dissimilar, the 4-and 6-year-olds were equally likely to confuse the source of circus acts that were unique to one story only, but 4-year-olds showed a tendency to perform more poorly than the 6year-olds for the specific details of acts that were common across stories.

Rather than considering storytellers as sources, many laboratory studies examining children's memory for repeated events consider each episode a separate source (e.g., 
Brubacher et al., 2011; Powell et al., 1999; Odegard et al., 2009). The similarity of the sources relies on the ratio of fixed, variable, and new details that are presented within the episodes. For example, a high proportion of fixed details means all episodes are quite similar while a high proportion of variable and new details means the episodes should be more discernible. The extant repeated-event research has presented children with inconsistent ratios of the detail-types. For example, Brubacher et al. (2011) included six variable and two new details per episode (in addition to six that alternated on a $75 \%-25 \%$ frequency), Roberts and Powell (2005) included eight fixed and eight variable details, and Connolly and Gordon (2014) included only variable details.

Comparison of different ratios of detail-types has not yet been experimentally manipulated within a single study to investigate the effect on children's episode reports. Instead, researchers have examined the similarity within a memory detail, rather than the similarity across occurrences caused by the composition of detail-types (e.g., Connolly \& Price, 2006; Howe, 2008; see also Brainerd, Reyna, \& Ceci, 2008). For example, Connolly and Price (2006) manipulated the degree of thematic similarity that memory details shared across episodes (e.g., puzzles always depicting an animal: a cow, tiger, pig, mouse, versus unrelated puzzles: an ant, grapefruit, \$5, socks). Following misinformation about a thematically-consistent alternative (e.g., elephant), older, but not younger, children were particularly suggestible for the thematically similar details. Connolly and Price's results may be explained by fuzzy-trace theory (FTT; Brainerd \& Reyna, 1990; 2004), which asserts that people encode a gist memory trace representing the overall patterns and meaning of an event and verbatim traces representing actual experiences at the event in parallel. Older children are more prone to construct and use gist (rather than verbatim) 
traces compared to young children (Brainerd et al., 2008), likely making them more susceptible to gist-consistent confusions.

\section{Current Study}

The current study was the first to systematically manipulate the ratio of detail-types to examine effects on children's episodic reports. To do this, we presented 5- to 9-year old children with a repeated event containing either a considerable proportion of fixed details (high similarity event) or a considerable proportion of variable and new details (low similarity event). The high similarity event was expected to foster strong script development, while the low similarity event was expected to foster a comparatively weaker script but the episodes (sources) would be more discernible. Accordingly, we predicted that children in the high similarity event would report proportionally more details related to the event script: fixed details $\left(\mathrm{H}_{1}\right)$, variable items (script slots) and variable instantiations (slotfillers, which are linked to an event script) $\left(\mathrm{H}_{2}\right)$, than children in the low similarity event. Children in the high similarity event were also expected to report proportionally more new details $\left(\mathrm{H}_{3}\right)$ because their stronger scripts should free-up cognitive resources to assist attending to these deviations from the script (Farrar \& Goodman, 1992). Since children struggle with source decisions when sources are similar (Lindsay et al., 1991), we predicted that children in the low similarity event would attribute more details to the correct episode (i.e., higher source accuracy) than children in the high similarity event $\left(\mathrm{H}_{4}\right)$.

We were also interested in children's ability to notice the similarity or discernibility of the episodes. Due to their stronger scripts for what usually occurs at the event, we expected children in the high similarity event to spontaneously mention similarities (i.e., 
fixed detail presentation patterns) and differences (i.e., variable detail presentation patterns) between the episodes proportionally more than children in the low similarity event $\left(\mathrm{H}_{5}\right)$.

\section{Method}

\section{Participants}

The sample was taken from a larger pool of data collected to examine the effects of interviewing techniques on children's recall of repeated events (see Danby, Brubacher, Sharman, \& Powell, 2015; 2017; Danby, Brubacher, Sharman, Powell, \& Roberts, 2017). Overall, 399 children aged 5- to 9-years-old were recruited from primary schools across Melbourne (Australia), and surrounding areas. They were randomly allocated to receive the high $(n=222)$ or low $(n=177)$ similarity event. Parents gave informed consent and children assented to participate.

To create the current sample, children who experienced the low similarity event were matched to children who experienced the high similarity event on: age (in years), gender, interview delay following the final episode of the event, and interview manipulations (such as practice narratives) until they could no longer be matched. The final sample comprised 152 children ( 76 girls, 76 boys), with 76 in each similarity condition. To explore developmental differences in children's memory reports, children were divided into age groups: younger ( $n=61,5-6$ years old; 31 girls, 30 boys) and older ( $n=91,7-9$ years old, 45 girls, 46 boys) children. Age group means were comparable in the high $\left(M_{\text {younger }}=5.61\right.$ years, $S D_{\text {younger }}=0.50 ; M_{\text {older }}=7.67$ years, $\left.S D_{\text {older }}=0.77\right)$ and low $\left(M_{\text {younger }}=5.70\right.$ years, $S D_{\text {younger }}=0.47 ; M_{\text {older }}=7.74$ years, $\left.S D_{\text {older }}=0.77\right)$ similarity conditions.

\section{Materials}


All children experienced a 25-minute scripted activity session (the Deakin Activities) on four occasions in groups of 20-30. Each group was randomly allocated to the high or low similarity condition. Each episode of the Deakin Activities comprised 16 target details administered in the same temporal order and centered around six main activities: meeting a puppet, listening to a story, doing a puzzle, relaxing, getting refreshed, and receiving a surprise. The event has been successfully employed in previous research (e.g., Brubacher et al., 2011).

The 16 details varied according to similarity condition. Children in the high similarity condition experienced nine fixed details presented identically on each episode (e.g., children did the same puzzle each time), six variable details that had different instantiations in each episode (e.g., children heard a different story each time), and one new detail in each episode did not appear any other time but was still consistent with the activities that could occur (e.g., children wore a badge in only one episode). Children in the low similarity condition experienced four fixed, nine variable, and three new details per episode. For each similarity condition, three counterbalanced presentation schedules were created such that fixed details for one group of children became variable or new for the next group. Children were randomly assigned to a counterbalanced version. There were no significant differences in children's total mentions of memory details, similarities, differences, or the accuracy of their reports across versions, $F \mathrm{~s} \leq 2.99, p \mathrm{~s} \geq .06$.

\section{Procedure}

The research was approved by the university's research ethics board. Trained research assistants conducted the Deakin Activities at children's schools twice a week for two weeks. Teachers were requested not to discuss the activities with their students. 
Individual interviews were conducted with each child five to eight days after the final episode by one interviewer.

After a brief rapport building phase where children provided an episodic practice narrative about something fun they had done recently (see Danby, Brubacher, Sharman, \& Powell, 2017), children were invited to talk about the Deakin Activities. The interviewer explained that she was naïve about the activities ("I wasn't there when you did the Deakin Activities, but I'd really like to know what happened"). Once children provided some information about the activities (verifying that they remembered them), the interviewer asked if the activities happened one time or more than one time. All children acknowledged that the activities occurred multiple times. The interviewer subsequently directed the children to nominate one well-remembered episode of the activities ("Tell me everything that happened the time you remember best”, see Danby, Brubacher, Sharman, Powell, \& Roberts, 2017 for information about this procedure) and prompted them to recall the episode in an open-ended, non-suggestive manner until they could not recall any further details. All children were then directed to select another episode ("Tell me everything that happened another time at the Deakin Activities") and were prompted in the same manner about the episode until they indicated that nothing more could be remembered. Interviews lasted approximately 20 minutes, and were audio-recorded and transcribed verbatim.

\section{Coding}

Initially, we analysed children's reports of the two recalled episodes separately. They showed similar patterns of results, however, so we collapsed them for simplicity (we present an average of the two recalled episodes). 
Reports of memory details. Each target detail that children reported was recorded, regardless of the particular episode in which it occurred, along with its frequency type (fixed, variable, and new). Details were then converted into proportions of the number of that detail-type present in that child's similarity condition. For example, if a child in the high similarity condition reported three fixed details, this was divided by nine (as the high similarity condition presented nine fixed details per episode) to give a proportion score of .33. For variable details, the specificity was also recorded: item reports referenced the broader regularly-occurring activity (e.g., "then we did a puzzle") and instantiation reports referenced the specific presentation during an episode (e.g., "one time we did a puzzle of a clown eating").

Accuracy. If children linked a variable instantiation or new detail to the episode, coders recorded whether it was accurately attributed. Accuracy scores for fixed details and variable item-level reports were not derived since these details occurred in the same way during each episode. Accuracy scores for variable instantiations and new details were converted into proportions of the number of the respective detail-type that the child had reported. For example, a child who reported four variable instantiations as occurring during an episode, but only one of the variable instantiations actually occurred in the episode, would have a variable instantiation accuracy score of .25 (i.e., 1 out of 4). Six children did not report any details unique to an episode of the activities, and thus did not have accuracy scores, but were included in all other analyses.

Mentions of differences and similarities. Coders recorded if children explicitly stated or listed differences (e.g., "we relaxed different body parts each time," "we relaxed our feet or our arms or our tummies each time") and similarities (e.g., "the badge was 
always the same") about the memory details. Reported differences were tallied and converted into proportions of the number of variable details in the episode, while similarities were converted into proportions of the number of fixed details in the episode. For example, if a child in the low similarity condition spontaneously mentioned that three details were different across occurrences and two details had stayed the same, these were divided by nine and four respectively (as these children experienced nine variable and four fixed details) to give proportional scores of .33 and .50. Children never mentioned similarities or differences with respect to new details, and they only reported differences (not similarities) associated with the variable details. All mentions of similarities and differences were correct, in that the details mentioned did indeed occur differently or similarly across each episode.

Reliability. Coding was completed on the pool of data $(n=399)$ from which the current sample was constructed. For reliability purposes, $15 \%$ of this pool was doublecoded; $5 \%$ at the outset and an additional $10 \%$ at the end of coding to ensure that coders maintained reliability. Kappa was calculated for identification of detail-types present in children's reports and their accuracy. Agreement ranged from $0.73-1.00(M=0.88)$. Percentage agreement for mentions of detail similarities and differences was $90.98 \%$ and $86.79 \%$ respectively. Disagreements were resolved through discussion.

\section{Results}

\section{Reports of memory details}

We first considered children's reports of fixed, variable, and new details, and children's accuracy using 2 (similarity condition: high, low) x 2 (age group: younger, older) 
ANOVAs ${ }^{1}$. Table 1 displays the means, standard deviations, and model statistics for all reports of memory details and children's accuracy.

Fixed details. There was a main effect of similarity condition, where children in the high similarity condition reported proportionally more fixed details than children in the low similarity condition, $F(1,148)=30.38, p<.001, \eta_{p}{ }^{2}=.17$. There was also a main effect of age group where older children reported proportionally more fixed details than younger children, $F(1,148)=26.12, p<.001, \eta_{p}{ }^{2}=.15$. The interaction was not significant, $F(1$, $148)=1.77, p=.19, \eta_{p}{ }^{2}=.01$.

Variable details. The ANOVA for variable details mentioned at an item-level was not significant. However, the ANOVA for variable details mentioned at an instantiationlevel showed only a main effect of age group where older children reported proportionally more variable instantiations than younger children, $F(1,148)=18.12, p<.001, \eta_{p}{ }^{2}=.11$. No other effects were significant, $F \mathrm{~s} \leq 2.68, p \mathrm{~s} \geq .10, \eta_{p}{ }^{2} \mathrm{~s} \leq .02$.

Variable Details Accuracy. Altogether 145 children $\left(n_{\text {high }}=72\right.$ [28 younger, 44 older], $n_{\text {low }}=73$ [27 younger, 46 older]) linked one or more variable instantiations to an episode to allow an accuracy score to be calculated. The ANOVA showed a main effect of similarity condition, where children in the low similarity condition were more accurate in their reports of variable instantiations than those in the high similarity condition, $F(1,141)$ $=5.582, p=.02, \eta_{p}{ }^{2}=.04$. There was also a main effect of age where older children were more accurate than younger children, $F(1,141)=22.37, p<.001, \eta_{p}{ }^{2}=.14$. The interaction was not significant, $F(1,141)=0.68, p=.41, \eta_{p}{ }^{2}=.005$.

New details. Only 70 children reported new details $\left(n_{\text {high }}=38[11\right.$ younger, 27 older], $n_{\text {low }}=32$ [12 younger, 20 older] $)$. The ANOVA $(n=152)$ showed a main effect for 
similarity condition, where children in the high similarity condition reported proportionally more new details than children in the low similarity condition, $F(1,148)=20.93, p<.001$,

$\eta_{p}{ }^{2}=.12$. There was also a main effect of age group; older children reported proportionally more new details than younger children, $F(1,148)=4.89, p=.03, \eta_{p}{ }^{2}=.03$. The interaction was not significant, $F(1,148)=2.17, p=.14, \eta_{p}{ }^{2}=.01$.

New Details Accuracy. Only 45 children $\left(n_{\text {high }}=19\right.$ [ 2 younger, 17 older], $n_{\text {low }}=26$ [10 younger, 16 older]) linked a new detail to an episode. Due to small cell sizes, ANOVA was not considered appropriate. A $t$-test showed that children in each similarity condition were equally accurate in their reports of new details, $t(43)=0.64, p=.53, d=0.20$.

\section{Mentions of differences and similarities}

Due to few children reporting differences or similarities, age differences were not examined. Altogether 79 children $\left(n_{\text {high }}=49\right.$ [18 younger, 31 older], $n_{\text {low }}=30$ [9 younger, 21 older]) made spontaneous reference to differences. The $t$-test $(n=152)$ showed that those in the high similarity condition mentioned proportionally more differences $(M=.10$, $S D=.12)$ than children in the low similarity condition $(M=.04, S D=.06), t(114.48)=$ $3.91, p<.001, d=.64$. Only 53 children $\left(n_{\text {high }}=27\right.$ [9 younger, 18 older], $n_{\text {low }}=26$ [8 younger, 18 older]) spontaneously mentioned similarities. A Mann-Whitney nonparametric test $(n=152)$ showed no significant differences in the proportional similarities mentioned under the high $($ mean rank $=72.78)$ and low (mean rank $=80.22)$ similarity conditions, $U=$ $2605(z=-1.23), p=.22, r=.10$.

\section{Discussion}

The degree of similarity between repeated event episodes affected children's autobiographical episode reports. Our findings are somewhat consistent with our 
expectations that children in the high similarity event would confirm and deploy a stronger script than children in the low similarity event. Children reported proportionally more fixed $\left(\mathrm{H}_{1}\right)$ and new $\left(\mathrm{H}_{3}\right)$ details after experiencing the high (rather than low) similarity event. The schema-confirmation deployment model asserts that with repeated experience children integrate the typically-occurring elements (e.g., fixed details) of an event into a script (Farrar \& Goodman, 1992). Once script elements are confirmed, the script is then deployed, freeing up cognitive resources to help the child attend to script deviations (e.g., new details). Since scripts are confirmed and deployed faster when the episodes are highly similar (Hudson et al., 1992), children in the high similarity condition likely had the opportunity to create stronger scripts than those in the low similarity condition, which in turn facilitated their reporting of fixed and new details.

Contrary to our expectations, children reported variable items and instantiations equally under both similarity conditions $\left(\mathrm{H}_{2}\right)$. Variable items were expected to form slots in children's scripts with variable instantiations linked to the script as slot-fillers. While this study has shown evidence consistent with the notion that children created stronger scripts after the high similarity event, those in the low similarity event may have still established scripts of their own. The low similarity event comprised primarily of variable details, so an event script would be focused around these (rather than fixed details, which were dominant in the high similarity event). Indeed, children initially exposed to variation in an event have been shown to then expect further variation, while those exposed to unchanging episodes expect more of the same (see Hudson et al., 1992 for review). In sum, we suggest that variable details were frequently recalled by both similarity conditions for different reasons; overall stronger scripts in the high similarity condition and a focus on predictable variation 
in the low similarity condition. Future investigation into children's ability to form scripts focused around predictable variation, rather than predictable similarity, would have theoretical as well as practical implications. Replicating the current study design but also asking children to recall their script (e.g., "What usually happens at Deakin Activities?") rather than individual episodes would shed further light on this idea.

As expected, children in the high similarity event fared worse than the low similarity event when monitoring which episode contained which particular variable instantiations $\left(\mathrm{H}_{4}\right)$. Monitoring sources is more difficult when sources are highly similar (Lindsay et al., 1991). The likeness of the high similarity episodes probably provided little opportunity for children to mentally differentiate them, thereby leading to reduced accuracy when attributing recalled variable instantiations to an episode. Fuzzy trace theory (FTT) asserts that memory for the specific happenings of an episode are contained within a unique verbatim trace for that episode (Brainerd \& Reyna, 1990; 2004). Retrieving the correct verbatim trace (i.e., for the correct episode) is likely more difficult when verbatim traces share more likenesses, and thus a more difficult task for those in the high, than low, similarity condition. Since they are never repeated, verbatim traces are likely weak for new details in both similarity conditions, contributing to poor reporting of these details overall.

Stronger scripts in the high similarity condition were expected to facilitate children's reporting of differences and similarities proportionally more than the low similarity condition $\left(\mathrm{H}_{5}\right)$. Similarly, according to FTT, the invariant structure of the high similarity event would be expected to facilitate children's construction of stronger gist traces (than the low similarity event) that support recall of differences and similarities. Similarities and differences would be contained within gist, rather than verbatim, traces 
because gist traces contain the overall structure and meaning of an event (e.g., "There are stories and animals that change each time"), rather than the episode-specific happenings (e.g., "the last time we heard a cat story") (Brainerd \& Reyna, 1990; 2004). In the current study, children in the high similarity condition mentioned differences, but not similarities, proportionally more than those in the low similarity condition. Differences may have been more salient to children than similarities, thus seeming more interesting and noteworthy to the children. Indeed, in an early examination of conversations between young children, Nelson and Gruendel (1979, p.81) noted children's propensity to list the differences they had experienced in scripted events, for example quoting a child's rendition of snack time as eating "hotdogs, or crackers, or cookies or something". Howe (2006) has further shown that children's memory for word-pairs is benefitted more by focusing on the differences between words rather than the similarities between them.

\section{Limitations and Future Research}

The high and low similarity events in the current study shared many commonalities outside the detail-type manipulation. For example, episodes in each condition were always completed in the children's classroom, by the same presenter, in the same temporal order, and were always learning-based activities. As such, the low similarity events were still quite routine and future research could consider increasing the distinctiveness (such has holding each episode in a different location) to test the impact on children's memory reports (see Howe, 2000). In the real world, repeated event episodes (sources) can range in similarity from being nearly identical (e.g., children's nightly bedtime routine) to being so dissimilar that they may not be considered a part of the same overarching event (e.g., going overseas versus going camping for vacation). The goal of the current study was to present 
events that were relatively routine but contained new details in each episode. As such, the findings may not necessarily generalise to situations where entire episodes (rather than details) are completely different to the typical event (e.g., Farrar \& Goodman, 1992).

\section{Conclusions}

The current study found that children's memory reports of repeated event episodes differed as a function of the similarity shared between episodes. Researchers have tested various interview techniques designed to improve the amount and accuracy of information that children report about repeated event episodes (see Brubacher et al., 2014), but they have not previously considered the ratio of detail-types comprising the to-be-remembered event. Investigating potential interactions with event composition when testing interview interventions will assist in understand the effects of such interventions. Given that children have been presented with varying ratios of detail-types across the extant research on repeated event memory, researchers should bear in mind the composition of the laboratory event used in past work when considering findings. 


\section{Footnote}

${ }^{1}$ The data were not normally distributed, so nonparametric Mann-Whitney U tests were conducted in parallel with ANOVAs and $t$-tests. All but one nonparametric test (mentions of similarities) showed the same pattern of results as the ANOVAs and $t$-tests. Given that ANOVAs and $t$-tests are relatively robust to violations of normality, we report the ANOVA and $t$-test results here for ease of interpretation except where the nonparametric result differed. 


\section{References}

Brainerd, C. J., \& Reyna, V. F. (1990). Gist is the grist: Fuzzy-trace theory and the new intuitionism. Developmental Review, 10, 3 - 47. doi:10.1016/0273-2297(90)90003$\mathrm{M}$

Brainerd, C. J., \& Reyna, V. F. (2004). Fuzzy-trace theory and memory development. Developmental Review, 24, 396 - 439. doi: 10.1016/j.dr.2004.08.005

Brainerd, C. J., Reyna, V. F., \& Ceci, S. J. (2008). Developmental reversals in false memory: A review of data and theory. Psychological Bulletin, 134, 343-382. doi:10.1037/0033-2909.134.3.343

Brubacher, S. P., Glisic, U., Roberts, K. P., \& Powell, M. (2011). Children's ability to recall unique aspects of one occurrence of a repeated event. Applied Cognitive Psychology, 25, 351 - 358. doi: 10.1002/acp.1696

Brubacher, S. P., Powell, M. B., \& Roberts, K. P. (2014). Recommendations for interviewing children about repeated experiences. Psychology, Public Policy, and Law, 20, 325 - 335. doi: 10.1037/law0000011

Connolly, D. A., \& Gordon, H. M. (2014). Can order of general and specific memory prompts help children to recall an instance of a repeated event that was different from the others? Psychology, Crime \& Law, 20, 852-864. doi:

$10.1080 / 1068316 x .2014 .885969$

Connolly, D. A., \& Lindsay, D. S. (2001). The influence of suggestions on children's reports of a unique experience versus an instance of a repeated experience. Applied Cognitive Psychology, 15, 205 - 223. doi: 10.1002/1099-

0720(200103/04)15:2<205::AID-ACP698>3.0.CO;2-F 
Connolly, D. A., \& Price, H. L. (2006). Children's suggestibility for an instance of a repeated event versus a unique event: The effect of degree of association between variable details. Experimental Child Psychology, 93, 207 - 223. doi:10.1016/j.jecp.2005.06.004

Danby, M. C., Brubacher S. P., Sharman, S. J., \& Powell, M. B. (2015). The effects of practice on children's ability to apply ground rules in a narrative interview. Behavioral Sciences and the Law, 33, 446 - 458. doi: 10.1002/bsl.2194

Danby, M. C., Brubacher S. P., Sharman, S. J., \& Powell, M. B. (2017). The effects of one versus two episodically oriented practice narratives on children's reports of a repeated event. Legal and Criminological Psychology, 22, 442 - 454. doi: 10.1111/lcrp.12110

Danby, M. C., Brubacher S. P., Sharman, S. J., Powell, M. B., \& Roberts, K. P. (2017). Children's reasoning about which episode of a repeated event is best remembered. Applied Cognitive Psychology, 31, 99-108. doi: 10.1002/acp.3306

Farrar, M. J., \& Goodman, G. S. (1992). Developmental changes in event memory. Developmental Review, 63, 173-187. doi: 10.2307/1130911

Fivush, R. (1984). Learning about school: The development of kindergartners' school scripts. Child Development, 55, 1697-1709. doi: 10.2307/1129917

Howe, M. L. (2000). The fate of early memories: Developmental science and the retention of childhood experiences. Washington, DC: American Psychological Association Howe, M. L. (2006). Developmental invariance in distinctiveness effects in memory. Developmental Psychology, 42, 1193-1205. doi:10.1037/0012-1649.42.6.1193 
Howe, M. L. (2008). Visual Distinctiveness and the Development of Children's False Memories. Child Development, 79, 65-79. doi:10.1111/j.1467-8624.2007.01111.x

Hudson, J., Fivush, R., \& Kuebli, J. (1992). Scripts and episodes: The development of event memory. Applied Cognitive Psychology, 6, 483 - 505. doi:

$10.1002 /$ acp. 2350060604

Hudson, J., \& Nelson, K. (1986). Repeated encounters of a similar kind: Effects of familiarity on children's autobiographic memory. Cognitive Development, 1, 253 271. doi: 10.1016/s0885-2014(86)80004-1

Johnson, M. K., Hashtroudi, S., \& Lindsay, D. S. (1993). Source monitoring. Psychological Bulletin, 114, 3 - 28. doi: 10.1037//0033-2909.114.1.3

Lindsay, D. S., Johnson, M. K., \& Kwon, P. (1991). Developmental changes in memory source monitoring. Journal of Experimental Child Psychology, 52, 297-318. doi: $10.1016 / 0022-0965(91) 90065-\mathrm{Z}$

Nelson, K., \& Gruendel, J. M. (1979). At morning it's lunchtime: A scriptal view of children's dialogues. Discourse Processes, 2, 73 - 94. doi: $10.1080 / 01638537909544456$

Odegard, T.N., Cooper, C.N., Lampinen, J.M., Reyna, V.F., \& Brainerd, C.J. (2009). Children's eyewitness memory for multiple real-life events. Child Development, 80 , 1977 - 1890. doi: 10.1111/j.1467-8624.2009.01373.x

Powell, M., Roberts, K., \& Guadagno, B. (2007). Particularisation of child abuse offences: Common problems when questioning child witnesses. Current Issues in Criminal Justice, 19, $64-74$. 
Powell, M. B., Roberts, K. P., Ceci, S. J., \& Hembrooke, H. (1999). The effects of repeated experience on children's suggestibility. Developmental Psychology, 35, 1462 1477. doi: 10.1037/0012-1649.35.6.1462

Powell, M. B., \& Thomson, D. M. (1996). Children's memory of an occurrence of a repeated event: Effects of age, repetition, and retention interval across three question types. Child Development, 67, 1988 - 2004. doi: 10.2307/1131605

Powell, M. B., Thomson, D. M., \& Dietze, P. M. (1997). Memories of separate occurrences of an event: Implications for interviewing children. Families in society: The journal of contemporary social services, 78, 600 - 608. doi:10.1606/1044-3894.3390

Roberts, K. P. (2002). Children's ability to distinguish between memories from multiple sources: Implications for the quality and accuracy of eyewitness statements. Developmental Review, 22, 403 - 435. doi: 10.1016/s0273-2297(02)00005-9

Roberts, K. P., \& Powell, M. B. (2001). Describing individual incidents of sexual abuse: a review of research on the effects of multiple sources of information on children's reports. Child Abuse \& Neglect, 25, 1643-1659. doi:10.1016/s0145-2134(01)002903

Roberts, K. P., \& Powell, M. B. (2005). Evidence of metacognitive awareness in young children who have experienced a repeated event. Applied Cognitive Psychology, 19, 1019 - 1031. doi: 10.1002/acp.1145

S. v R. (1989). 168 CLR 266.

Schank, R.C., \& Abelson, R.P. (1977). Scripts, plans, goals, and understanding: An inquiry into human knowledge structures. Hillsdale, NJ: Lawrence Erlbaum Associates. 
Slackman, E., \& Nelson, K. (1984). Acquisition of an unfamiliar script in story form by young children. Child Development, 55, 329-340. doi:10.2307/1129946

Thierry, K. L., \& Pipe, M.-E. (2009). The susceptibility of young preschoolers to source similarity effects: Confusing story or video events with reality. Journal of Experimental Child Psychology, 102, 392 - 407. doi: 10.1016/j.jecp.2008.12.005 
Table 1

Descriptive and model statistics

\begin{tabular}{|c|c|c|c|c|c|c|c|c|c|c|c|}
\hline & \multicolumn{4}{|c|}{ Similarity Condition } & \multicolumn{4}{|c|}{ Age Group } & & & \\
\hline & \multicolumn{2}{|c|}{ High } & \multicolumn{2}{|c|}{ Low } & \multicolumn{2}{|c|}{ Younger } & \multicolumn{2}{|c|}{ Older } & \multicolumn{3}{|c|}{ Model statistics } \\
\hline & $M$ & $S D$ & $M$ & $S D$ & $M$ & $S D$ & $M$ & $S D$ & $F(3,148)$ & $p$ & $\eta_{p}^{2}$ \\
\hline \multicolumn{12}{|l|}{ Amount reported } \\
\hline Fixed & .41 & .20 & .24 & .20 & .24 & .14 & .39 & .23 & 20.62 & $<.001$ & .30 \\
\hline Variable item & .24 & .11 & .23 & .12 & .24 & .11 & .23 & .12 & 0.68 & .57 & .01 \\
\hline Variable instantiation & .38 & .27 & .32 & .17 & .26 & .23 & .41 & .20 & 7.27 & $<.001$ & .13 \\
\hline New & .33 & .38 & .10 & .16 & .15 & .26 & .28 & .33 & 10.48 & $<.001$ & .18 \\
\hline Accuracy of reported de & etails & & & & & & & & $F(3,141)$ & $p$ & $\eta_{p}^{2}$ \\
\hline Variable instantiation & .23 & .25 & .34 & .25 & .17 & .21 & .36 & .25 & 10.16 & $<.001$ & .18 \\
\hline New & .44 & .44 & .37 & .41 & - & - & - & - & - & - & - \\
\hline
\end{tabular}

